\title{
A Rare Case of Acute Bilateral Endothelial Decompensation after Prophylactic Nd:YAG Laser Iridotomy Requiring Endothelial Keratoplasty
}

\author{
Yishay Weill ${ }^{1}$, Adi Abulafia ${ }^{2}$, David Smadja ${ }^{3}$, Eduardo Roditi ${ }^{4}$, Joel Hanhart ${ }^{5}$, David Zadok ${ }^{6}$
}

\begin{abstract}
Aim: To describe a case of acute bilateral endothelial decompensation following prophylactic Nd:YAG laser iridotomy (LI) for occludable angles. Background: Although regarded safe, LI can occasionally be a source of various ocular complications, including corneal endothelial damage. In the herein case, we describe the first case of acute bilateral endothelial decompensation after Nd:YAG LI.

Case description: A 63-year-old man was referred for consultation due to visual acuity deterioration in both eyes 2 weeks after undergoing an uneventful prophylactic LI for occludable angles. On examination, bilateral corneal edema with Descemet's membrane folds was observed. Direct corneal damage from the laser beam was not seen. Specular microscopy failed to count endothelial density. Anterior-segment optical coherence tomography (OCT), ultrasound biomicroscopy, and ocular biometry were performed. The patient was referred for bilateral endothelial keratoplasty.

Conclusion: Subacute endothelial dysfunction should be considered as a possible adverse event following Nd:YAG LI and patients should be advised accordingly.

Clinical relevance: Surgeons should be aware of the potentially devastating complication of bilateral corneal decompensation following routine $\mathrm{Nd}$ :YAG LI, even in patients without preexisting corneal injury. Patients should be advised accordingly.

Keywords: Angle-closure glaucoma, Corneal edema, Corneal transplant, Laser iridotomy, Nd:YAG laser.

Journal of Current Glaucoma Practice (2020): 10.5005/jp-journals-10078-1285
\end{abstract}

\section{BACKGROUND}

Laser iridotomy (LI) is a well-established procedure for the treatment and prevention of angle-closure glaucoma (ACG). Although both argon and neodymium-doped yttrium aluminum garnet (Nd:YAG) $\mathrm{LI}$ are regarded as being safe, they carry the risks of causing various ocular complications, including elevated intraocular pressure (IOP), iritis, hemorrhage, lens opacity, and corneal burns. ${ }^{1}$ Decreased endothelial density after LI has been reported in a few long-term studies. ${ }^{2}$ Late endothelial decompensation was documented in patients after argon $\mathrm{LI}$, eventually necessitating corneal transplantation years after the procedure. ${ }^{2}$ To the best of our knowledge, acute endothelial decompensation after $\mathrm{Nd}$ :YAG LI has not been reported before.

\section{Case Description}

A 63-year-old man was referred to our corneal unit 2 weeks after undergoing an uneventful prophylactic bilateral Nd:YAG LI for occludable angles. He complained of bilateral deterioration of visual acuity (VA) that began 3 days following the procedure. His surgical records described the Nd:YAG LI as being uneventful, with normal postoperative IOP and no hyphema or intraocular hemorrhage. Following the procedure, he was treated with topical $0.1 \%$ dexamethasone four times a day for 1 week. His medical history included benign prostatic hypertrophy treated with an $\mathrm{a}_{1-}$ adrenergic receptor blocker and an ocular history that included high hyperopia and mild nuclear sclerosis cataract. He did not complain of decreased vision before the Nd:YAG LI procedure, and there was no personal or familial history of fluctuating vision, episodes of ocular pain, corneal dystrophy, or corneal transplantation.

\begin{abstract}
${ }^{1-6}$ Department of Ophthalmology, Shaare Zedek Medical Center, Affiliated to the Hebrew University, Jerusalem, Israel

Corresponding Author: Yishay Weill, Department of Ophthalmology, Shaare Zedek Medical Center, Affiliated to the Hebrew University, Jerusalem, Israel, Phone: +972-2-6555246, e-mail: yishayweill@gmail. com

How to cite this article: Weill Y, Abulafia A, Smadja D, et al. A Rare Case of Acute Bilateral Endothelial Decompensation after Prophylactic Nd:YAG Laser Iridotomy Requiring Endothelial Keratoplasty. J Curr Glaucoma Pract 2020;14(3):109-111.
\end{abstract}

Source of support: Nil

Conflict of interest: None

He underwent an ophthalmic examination in our clinic, and it revealed a corrected VA of RE 20/60, LE 20/40 (glasses +7.50 D-0.75 D $\times 21$ and +8.50 D-1.75 D $\times 92$ right eye and left eye, respectively). The IOP was $12 \mathrm{~mm} \mathrm{Hg}$ in both eyes. Slit-lamp examination revealed bilateral diffuse corneal edema with Descemet's membrane folds and a few epithelial bullae. The corneal edema was worse in the right eye, and inferior predominance edema was detected in the left eye (Fig. 1). There were no signs of guttae, Descemet's membrane detachment, corneal opacities, or focal corneal damage. The rest of the examination demonstrated shallow and quiet anterior chambers, bilateral normal-sized and transilluminated superotemporal peripheral iridotomies, round and reactive pupils, and mild nuclear sclerosis cataract. The fundus examination ruled out any definitive changes in the retina. 

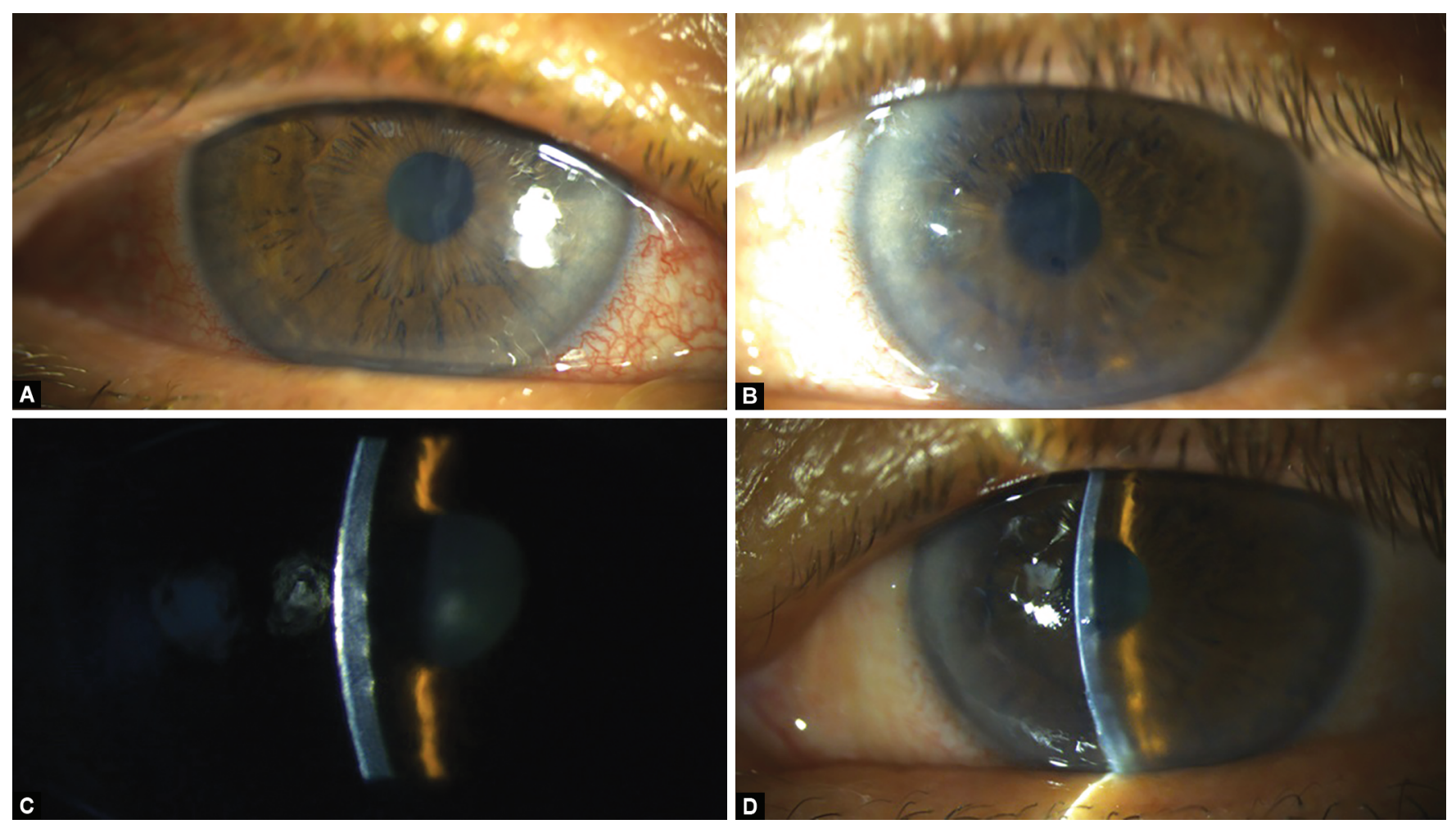

Figs $1 \mathrm{~A}$ to D: Right eye (A and $C$ ) and left eye (B and D) anterior segment photographs showing diffuse corneal edema and Descemet's membrane folds

Non-touch specular microscopy (EM-3000, Tomey, Japan) failed to measure endothelial density in both eyes. Anterior segment spectral-domain optical coherence tomography (OCT) (HeidelbergSpectralis, version 6.9.4.0, Heidelberg Engineering, Germany) demonstrated an attached Descemet's membrane with prominent folds in each eye (Fig. 2), and ultrasound biomicroscopy confirmed the presence of narrow angles. The axial length was 20.45 and 20.17 $\mathrm{mm}$, the anterior chamber depth was 2.10 and $2.25 \mathrm{~mm}$, and the central corneal thickness was 736 and $689 \mu \mathrm{m}$ in the right and left eyes, respectively (Swept-Source OCT Biometer, IOLMaster 700, Carl Zeiss, Germany).

The patient was diagnosed as having acute corneal decompensation following Nd:YAG LI. He was treated with topical $0.1 \%$ dexamethasone and $\mathrm{NaCl} 5 \%$ ointment for 2 months without improvement and was subsequently referred for bilateral endothelial keratoplasty.

\section{Discussion}

Peripheral LI is a well-established procedure for ACG and one that is widely performed worldwide. Potential cornea-related complications following $\mathrm{LI}$ are numerous, and they may include endothelial damage, which ultimately can lead to endothelial decompensation necessitating corneal transplantation. ${ }^{1}$ Several mechanisms of LI-related endothelial damage have been proposed, including direct corneal burn, thermal and shockwave injury, shear stress, iris pigment dispersion, and blood-aqueous barrier disruption. ${ }^{2,3}$ Although some risk factors predicting significant endothelial cell loss after LI have been hypothesized, no direct association has been found between any single factor and the development of corneal decompensation. ${ }^{2}$
In the few studies that described endothelial decompensation after LI, the symptoms had appeared years after the procedure, and argon laser rather than Nd:YAG laser had been applied. ${ }^{2}$ Argon $\mathrm{LI}$ is achieved by means of a photocoagulation mechanism, while $\mathrm{Nd}$ :YAG laser pierces the iris through photodisruption. ${ }^{2}$ Endothelial cell density was shown to decrease after both procedures, although to a less extent after NG:YAG laser. ${ }^{4}$ Part of the reason for that difference may be that penetration of the iris with the Nd:YAG laser generally requires fewer laser spots than an argon laser due to its high-power density. ${ }^{2,5,6}$

In the current case report, our patient developed bilateral endothelial decompensation 2 weeks after a documented uneventful prophylactic Nd:YAG LI for occludable angles. This sequela is extremely unusual in light of the rapid progression of corneal decompensation, the application of an Nd:YAG laser rather than an argon laser, and the fact that the patient had no history of corneal pathology. The underlying etiology for the presented rare occurrence was not determined. Acute inflammation, a high IOP, or pigment dispersion were not likely to contribute to the corneal damage since the IOP was normal at presentation as well as after the procedure, the anterior chamber was clear, and no pigment deposition was observed on the endothelium. Direct corneal damage was also ruled out since none was documented following the $\mathrm{LI}$, no signs of damage were observed on presentation, and because the most injured area did not correspond to the LI location. Lastly, the absence of corneal dystrophy findings, as well as the lack of prior visual symptoms, indicates that it was highly unlikely for preexisting corneal dystrophy to contribute to the acute corneal decompensation. Although we cannot rule out non-guttate Fuchs endothelial corneal dystrophy (FED) in our patient, ${ }^{7}$ the absence of any clinical signs or symptoms before and following the $\mathrm{LI}$ and the 

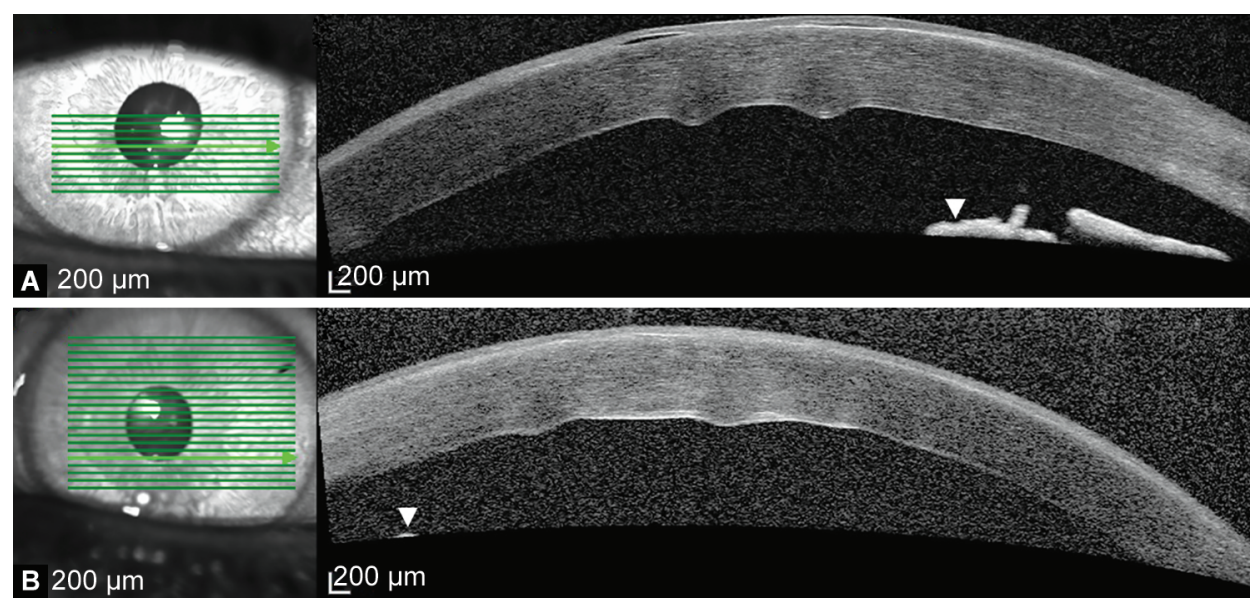

Figs 2A and B: Anterior-segment optical coherence tomography (Spectralis, Heidelberg Engineering, Heidelberg, Germany) of the right eye (A) and left eye (B) demonstrating corneal edema with Descemet's membrane folds bilaterally and bullae in the right eye. Note the location of the iris stroma (arrowheads), indicating narrow angles

lack of predominant central corneal edema makes FED an unlikely diagnosis.

Recent studies have questioned the suitability of $\mathrm{LI}$ as a firstline treatment for primary ACG (PACG) in a patient with normal IOP and suggested that cataract extraction with IOL implantation may serve as a superior alternative. ${ }^{8,9}$ While $\mathrm{LI}$ solely resolves the relative pupillary block component in ACG, phacoemulsification with IOL implantation deepens the anterior chamber by a posterior repositioning of the ciliary processes due to a debulking effect, resulting in widening of the anterior chamber angle. ${ }^{10}$ It has also been suggested that cataract extraction may have lower failure rates in lowering IOP in the long-term. ${ }^{2}$ The recent randomized controlled EAGLE study concluded that clear lens extraction had better efficacy and was more cost-effective than LI for PACG. ${ }^{8,9}$ Another possible reason for favoring phacoemulsification in PACG patients is the effect of the procedures on the corneal endothelium, with several studies have demonstrated a lower rate of endothelial cell loss after phacoemulsification. ${ }^{2}$ Although larger trials are still needed to better define the comparative effect of these two interventions on corneal endothelium health, performing phacoemulsification rather than $\mathrm{LI}$ in our patient may have prevented the devastating corneal injury.

\section{ConcLusion}

Laser iridotomy can impair VA due to rapid corneal endothelial decompensation, in our case without a detectable etiology. To the best of our knowledge, this is the first to report acute bilateral corneal decompensation following Nd:YAG LI necessitating keratoplasty. Although more cases are needed to reinforce this observation, physicians should be aware of this potential sightthreatening complication, and patients should be informed accordingly.

\section{Clinical Significance}

Although considered a simple and straightforward procedure, Nd:YAG LI can be a cause of various ocular complications. In this context, surgeons should be aware of the rare and potentially devastating complication of bilateral corneal decompensation following routine $\mathrm{Nd}$ :YAG LI, necessitating keratoplasty, even in patients without preexisting corneal injury, and advise their patients accordingly.

\section{References}

1. Radhakrishnan S, Chen PP, Junk AK, et al. Laser peripheral iridotomy in primary angle closure: A report by the American Academy of Ophthalmology. Ophthalmology. 2018;125(7):1110-1120. DOI: 10.1016/j.ophtha.2018.01.015.

2. Wang PX, Koh VTC, Loon SC. Laser iridotomy and the corneal endothelium: A systemic review. Acta Ophthalmol 2014;92(7):604616. DOI: $10.1111 /$ aos.12367.

3. Kaji Y, Oshika T, Usui T, et al. Effect of shear stress on attachment of corneal endothelial cells in association with corneal endothelial cell loss after laser iridotomy. Cornea. 2005;24(8 SUPPL.):55-58. DOI: 10.1097/01.ico.0000178735.27674.52.

4. Robin AL, Pollack IP. A comparison of neodymium:YAG end argon laser iridotomies. Ophthalmology. 1984;91(9):1011-1016. DOI: 10.1016/ S0161-6420(84)34199-9.

5. Tomey KF, Shammas IV, Traverso CE. Neodymium-YAG laser iridotomy in the treatment and prevention of angle closure glaucoma. Arch Ophthalmol 1987;105(4):476-481. DOI: 10.1001/arc hopht.1987.01060040046029.

6. Moster MR, Schwartz LW, Spaeth GL, et al. Iridectomy: A controlled study comparing argon and neodymium:YAG. Ophthalmology. 1986;93(1):20-24. DOI: 10.1016/S0161-6420(86)33787-4.

7. Adamis AP, Filatov V, Tripathi BJ, et al. C. Fuchs' endothelial dystrophy of the cornea. Surv Ophthalmol 1993;38(2):149-168. DOI: 10.1016/0039-6257(93)90099-S.

8. Azuara-Blanco A, Burr J, Ramsay C, et al. Effectiveness of early lens extraction for the treatment of primary angle-closure glaucoma (EAGLE): a randomised controlled trial. Lancet. 2016;388(10052):13891397. DOI: 10.1016/S0140-6736(16)30956-4.

9. Tanner L, Gazzard G, Nolan WP, et al. Has the EAGLE landed for the use of clear lens extraction in angle-closure glaucoma? And how should primary angle-closure suspects be treated? Eye. 2020;34(1):40-50. DOI: 10.1038/s41433-019-0634-5.

10. Melese E, Peterson JR, Feldman RM, et al. Comparing laser peripheral iridotomy to cataract extraction in narrow angle eyes using anterior segment optical coherence tomography. PLoS One 2016;11(9):1-16. DOI: 10.1371/journal.pone.0162283. 